\title{
Rotatory quantization of charge-conjugation symmetric systems. 1. Harmonic oscillators.
}

\begin{abstract}
Zahid Zakir $^{1}$
Abstract

In a system of a particle and antiparticle in the harmonic potential, represented as an oscillator with a complex generalized coordinate, there is a global U(1) symmetry and the charge conjugation (C) symmetry. It is shown that two pairs of ladder operators, introduced at the frequency decomposition of canonical variables, are not mutually charge-conjugate and that therefore their standard interpretation as operators of the charge-conjugate quanta breaks C-symmetry. Operator identities between bilinear products of the ladder operators are discovered, allowing expressing observables through charge-conjugate operators and it is correct to take into account Csymmetry. It is shown that these identities are maintained and at insert of the Csymmetric interactions. In a Lagrangian unsymmetrized and symmetrized orderings of complex conjugate operators of a momentum lead to different charge operators and are not equivalent at interaction with the gauge field. It is shown that due to C-symmetry conditions a zero-point charge does not arise in both orderings and in the first case a zero-point energy disappears also. The contribution of interaction with the gauge field and anharmonic potentials in higher orders of perturbation theory is considered. The same system also can be presented as a particle with positive and negative frequencies and, if to consider that a sign of mass of the particle coincides with a sign of its frequency, then the norm of negative frequency states remains positive.
\end{abstract}

PACS: 03.65.Ge, 11.30.Er, 1130.Ly, 11.90. + t

Key words: Hamiltonian dynamics, discrete symmetries, quantization

\section{Content}

Introduction

1. Oscillating particle and antiparticle: charge conjugation symmetry and its violation ...............16

2. A charge conjugation symmetric system of oscillators with and without a zero-point energy ..18

3. About physical consequences of the charge conjugation symmetry of oscillators ………..........20

4. Interactions of charge conjugation symmetric oscillators ........................................................23

5. The problem of negative norm for negative-frequency states and its solution .........................24

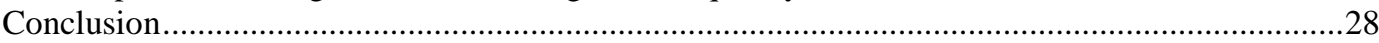

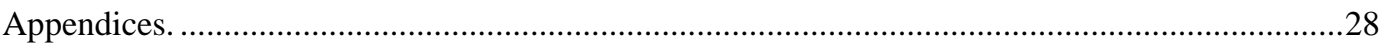

1. The uncertainty relations for non-hermitian variables .........................................................2

2. Canonical formalism for systems with complex variables ...................................................29

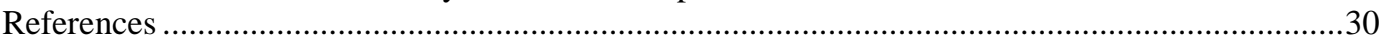

${ }^{1}$ Centre for Theoretical Physics and Astrophyics, Tashkent, Uzbekistan; zahidzakir@theor-phys.org 


\section{Introduction}

In the standard theory of linear and circular harmonic oscillators, despite it would seems studied in details, there are some problems long time remained unsolved. Their recently found solutions [1-3] have appeared natural, and their consequences - nontrivial.

In the standard formulation of the quantum field theory the normal modes of fields are quantized by analogy to linear harmonic oscillators and consequently some problems in the field theory have appeared as consequences of the problems in the oscillatory theory only and also have been simply solved.

The first problem is the prediction of a divergent zero-point vacuum energy for quantum fields that due to the existence of gravitation leads to inconsistency of such theories for this reason only. The zero-point fluctuations, clearly, are inevitable for linear harmonic oscillators. The answer to a question on, can the symmetries of systems of the same type oscillators lead to elimination of zero-point energy, seemed clear and negative. Therefore, it has been accepted that the zero-point energies are inevitable and for their elimination a set of modifications of foundations of quantum mechanics and field theory have been proposed.

The second problem is the negative norm of negative-frequency states of an oscillator. Such norm leads to negative probabilities and makes the theory physically inconsistent. These states can be neglected in the nonrelativistic theory, but they will appear in relativistic theories. Many proposals for solution of the problem are wellknown and are based on a set of modifications of the foundation of quantum mechanics.

The recently found solution to both problems [1-3] within the frameworks of the standard quantum mechanics had based on two new ideas - one a methodological and one a physical. The first idea is that the standard treatment probably contains certain hidden unphysical restrictions ("virus hypotheses") which lead to well-known unphysical consequences. The presence and removal of these unnecessary restrictions then will lead to the states with positive norm and to relativistic systems without zero-point energy. Therefore, instead of the complicating the theory by new hypotheses the problem has been put to find and exclude unnecessary hypotheses of the present theory that is more simple and solvable problem.

The second idea is that revealing of such hypotheses of the standard approach should begin on checking of artificial restrictions on external parameters of the theory and obeying to restrictions of the basic discrete symmetries, such as spacetime inversion and the charge conjugation symmetry.

In the present paper a systematic description of such approach and obtained results are presented. There shortly considered the restrictions from the symmetries, found the statements breaking them and, excepting the last ones, the standard theory of the harmonic oscillator is reformulated in more consecutive form. The norms of all states thus are positive and it is shown that in the systems of harmonic oscillators with charge conjugation symmetry the zero-point energy does not arise.

In the sections 1-3 the charge conjugation symmetric systems of oscillators are considered. In section 4 interactions in such systems of oscillators are considered. In the section 5 the solution of the negative norm problem for negative-frequency states is given. Main references to the literature have been presented in the previous publications. 
16 Zakir Z. (2011) Theoretical Physics, Astrophysics and Cosmology, 6, 14; TPAC: 3900-021 v2.

\section{Oscillating particle and antiparticle: charge conjugation symmetry and its violation}

Let's consider a charge conjugation symmetric system containing a particle and an antiparticle in the linear harmonic oscillatory potential along the same axis $x$ and the violation of this symmetry at quantization. In its own variables the antiparticle, as well as the particle, is described by a positive-frequency oscillator. Dissimilarity from the particle in a sign of the charge only leads to the charge conjugation symmetry, while the charge is exhibited only at inserting of interactions.

The Lagrangian then looks like:

$$
L=\frac{m}{2}\left[\left(\partial_{t} x_{1}\right)^{2}+\left(\partial_{t} x_{2}\right)^{2}-\omega^{2}\left(x_{1}^{2}+x_{2}^{2}\right)\right],
$$

where $x_{1}$ and $x_{2}$ are coordinates of the particle and antiparticle. It leads to the momenta $p_{j}=m \partial_{t} x_{j}(j=1,2)$ and the Hamiltonian:

$$
H=\frac{1}{2 m}\left[p_{1}^{2}+p_{2}^{2}+(m \omega)^{2}\left(x_{1}^{2}+x_{2}^{2}\right)\right] .
$$

Equations of motion lead to the frequency decompositions:

$$
x_{j}=\frac{1}{\sqrt{2 m \omega}}\left(a_{j} e^{-i \omega t}+a_{j}^{*} e^{i \omega t}\right), \quad p_{j}=\frac{-i m \omega}{\sqrt{2 m \omega}}\left(a_{j} e^{-i \omega t}-a_{j}^{*} e^{i \omega t}\right),
$$

and the inverse formulas look like:

$$
a_{j}=\frac{e^{i \omega t}}{\sqrt{2 m \omega}}\left(m \omega x_{j}+i p_{j}\right), \quad a_{j}^{*}=\frac{e^{-i \omega t}}{\sqrt{2 m \omega}}\left(m \omega x_{j}-i p_{j}\right) .
$$

The commutators give:

$$
i\left[p_{j}, x_{j}\right]=1 \Rightarrow\left[a_{j}, a_{j}^{*}\right]=1,
$$

and the Hamiltonian in terms of the ladder operators takes a form:

$$
H=\omega\left(a_{1}^{*} a_{1}+a_{2}^{*} a_{2}+1\right) \text {. }
$$

Lagrangian (1) of the system of two oscillating charge-conjugate particles is symmetrical under a rotation on a configurationally plane of the variables $\left(x_{1}, x_{2}\right)$, leaving unchanged a "generalized radius" $r=\left(x_{1}^{2}+x_{2}^{2}\right)^{1 / 2}$. The symmetries of the system can be revealed more explicitly at a complex representation of these variables:

$$
\begin{array}{ll}
q=\frac{1}{\sqrt{2}}\left(x_{1}+i x_{2}\right), & q^{*}=\frac{1}{\sqrt{2}}\left(x_{1}-i x_{2}\right), \\
p=\frac{1}{\sqrt{2}}\left(p_{1}-i p_{2}\right), & p^{*}=\frac{1}{\sqrt{2}}\left(p_{1}+i p_{2}\right) .
\end{array}
$$

The frequency decompositions then take a form:

$$
\begin{aligned}
& q=\frac{1}{\sqrt{2 m \omega}}\left(a e^{-i \omega t}+\beta^{*} e^{i \omega t}\right), \quad q^{*}=\frac{1}{\sqrt{2 m \omega}}\left(a^{*} e^{i \omega t}+\beta e^{-i \omega t}\right), \\
& p=\frac{i m \omega}{\sqrt{2 m \omega}}\left(a^{*} e^{i \omega t}-\beta e^{-i \omega t}\right), \quad p^{*}=\frac{-i m \omega}{\sqrt{2 m \omega}}\left(a e^{-i \omega t}-\beta^{*} e^{i \omega t}\right),
\end{aligned}
$$

where the new ladder operators are related with former ones as: 


$$
\begin{aligned}
a & =\frac{1}{\sqrt{2}}\left(a_{1}+i a_{2}\right), \quad \beta=\frac{1}{\sqrt{2}}\left(a_{1}-i a_{2}\right), \\
a^{*} & =\frac{1}{\sqrt{2}}\left(a_{1}^{*}-i a_{2}^{*}\right), \quad \beta^{*}=\frac{1}{\sqrt{2}}\left(a_{1}^{*}+i a_{2}^{*}\right) .
\end{aligned}
$$

In a system with a complex canonical variable $q$ to the rotational symmetry on the complex plane there corresponds a conserved charge, the operator of which is:

$$
Q=i\left(q^{*} p^{*}-p q\right)=a^{*} a-\beta \beta^{*},
$$

This operator provides an interaction of quanta with an external field and in such systems there is a charge-conjugation $(\mathrm{C})$ symmetry.

While it is necessary to consider the ladder operators $\beta, \beta^{*}$ as "auxiliary" ones, since, as it will be shown further, they are not "charge-conjugate" ones to the operators $a, a^{*}$ of the "basic" quanta [1,2]. If in this system the charge conjugation symmetry is exactly holds, $\beta, \beta^{*}$ should be related with the operators $b, b^{*}$, initially introduced as a "charge-conjugate" ones to $a, a^{*}$, only by using the symmetry conditions.

The commutators $i[p, q]=i\left[p^{*}, q^{*}\right]=1$ give the commutators for the ladder operators, nonzero of which are:

$$
\left[a, a^{*}\right]=\left[\beta, \beta^{*}\right]=1 .
$$

The Hamiltonian now takes a form:

$$
H=\frac{1}{m} p p^{*}+m \omega^{2} q^{*} q=\omega\left(a^{*} a+\beta \beta^{*}\right)
$$

In this Hamiltonian and the charge operator (10) it has been accepted to rearrange the second pair of operators in a normal ordered form $\beta \beta^{*}=\beta^{*} \beta+1$ by using the commutator and then, as well as in (6), there appeared the zero-point energy for two types of oscillators.

However, the charge conjugation symmetry, which formally takes place before quantization, appears as broken by quantum correction - by a ground state zero-point charge. This situation has some features of a quantum anomaly (symmetry is broken by the quantum correction), and spontaneous symmetry breaking (the Hamiltonian is symmetrical, while the ground state does not).

At lack of an external field there is degeneration of energy levels under two values of the charge. The charge conjugation operation $C$, at which energy of the system does not change, while the charge changes a sign, is defined in the form:

$$
H^{c} \equiv C H C^{-1}=H, \quad Q^{c} \equiv C Q C^{-1}=-Q .
$$

The ladder operators, charge-conjugate to former ones, are defined as:

$$
\begin{gathered}
b=C a C^{-1}, \quad b^{*}=C a^{*} C^{-1}, \quad \alpha=C \beta C^{-1}, \quad \alpha^{*}=C \beta^{*} C^{-1} . \\
{\left[b, b^{*}\right]=\left[\alpha, \alpha^{*}\right]=1 .}
\end{gathered}
$$

If, as it has been accepted earlier, to identify the operators:

$$
b \rightarrow \beta, \quad b^{*} \rightarrow \beta^{*},
$$

considering $\beta, \beta^{*}$ as charge-conjugate to $a, a^{*}$, this will lead to the direct violation of the charge conjugation symmetry: 
18 Zakir Z. (2011) Theoretical Physics, Astrophysics and Cosmology, 6, 14; TPAC: 3900-021 v2.

$$
\begin{aligned}
& Q=a^{*} a-b b^{*}=a^{*} a-b^{*} b-1, \\
& Q^{c}=b^{*} b-a a^{*}=-\left(a^{*} a-b^{*} b-1\right)-2=-Q-2 .
\end{aligned}
$$

Here in the charge operator there is a ground state "zero-point charge", but the sign of this constant does not change at the charge conjugation and one of requirements of the charge conjugation symmetry (13) will be broken: $Q^{c} \neq-Q$. This violation follows from the identification (16) and "abnormal" product of operators $\beta \beta^{*}$ in (10) and (12).

Thus, the implicit hypotheses of the former standard treatment incompatible with the symmetries of the system, are two statements that: 1) the operators $\beta, \beta^{*}$ are chargeconjugate to $\left.a, a^{*} ; 2\right)$ the definition of the "normal" product, accepted for the operators $a^{*} a$ of the basic quanta, is applicable for the operators $\beta, \beta^{*}$ also.

In fact in the system with exact charge conjugation symmetry the obeying to the restrictions of this discrete symmetry changes the situation and at least one of these two statements becomes unphysical. Their elimination and how it will recover the charge conjugation symmetry will be considered in the next section.

\section{A charge conjugation symmetric system of oscillators with and without a zero-point energy}

Formally it is possible to recover the charge conjugation symmetry in the system of oscillating particle and antiparticle simply by redefining the observables:

$$
\tilde{H}=\frac{1}{2}\left(H+H^{c}\right), \quad \tilde{Q}=\frac{1}{2}\left(Q-Q^{c}\right) .
$$

when the Hamiltonian remains as former one, but the charge transfers into:

$$
\tilde{Q}=\frac{1}{2}\left(a^{*} a+a a^{*}-b^{*} b-b b^{*}\right)=a^{*} a-b^{*} b=-\tilde{Q}^{c} .
$$

But, it means an initial choice of a charge-conjugation symmetric Lagrangian containing instead of $Q$ an operator $\tilde{Q}$, which means already other model Lagrangian $\tilde{L}$. At inserting of interactions it leads to physically different consequences, in particular, from (19) we see that in the ground state there is no zero-point charge.

Further we will consider models without a special symmetrization, i.e. with "minimal" Lagrangians $L$, and the relations, linking operators $\beta$ and $b$ we will obtain from the charge-conjugation symmetry requirements $[1,2]$.

The Hamiltonian and the charge operator of the states, charge-conjugate to former ones, then take a form:

$$
H^{c}=\omega\left(b^{*} b+\alpha \alpha^{*}\right), \quad Q^{c}=b^{*} b-\alpha \alpha^{*} .
$$

The $C$-symmetry requirements (13) in a detailed form represent the relations:

$$
\begin{aligned}
& H=\omega\left(a^{*} a+\beta \beta^{*}\right)=\omega\left(b^{*} b+\alpha \alpha^{*}\right)=H^{c}, \\
& Q=a^{*} a-\beta \beta^{*}=-\left(b^{*} b-\alpha \alpha^{*}\right)=-Q^{c},
\end{aligned}
$$

giving:

$$
\begin{aligned}
& a^{*} a+\beta \beta^{*}=b^{*} b+\alpha \alpha^{*}, \\
& a^{*} a-\beta \beta^{*}=-b^{*} b+\alpha \alpha^{*} .
\end{aligned}
$$

By adding and subtracting these two relations, we find two identities between the operator products: 


$$
\alpha \alpha^{*}=a^{*} a, \beta \beta^{*}=b^{*} b .
$$

The second identity here is the charge-conjugate form of the first one.

These identities allow excluding completely from the Hamiltonian and the charge operator the auxiliary operators $\beta, \beta^{*}$ and $C$-conjugate them operators $\alpha, \alpha^{*}$, by expressing then through the operators $a, a^{*}$ of the basic quanta and the charge-conjugate them operators $b, b^{*}$ :

$$
\begin{aligned}
& H=H^{c}=\omega\left(a^{*} a+b^{*} b\right), \\
& Q=-Q^{c}=a^{*} a-b^{*} b .
\end{aligned}
$$

As we see, the operators are naturally normal ordered and, consequently, the systems with complex canonical variables and with exact $C$-symmetry do not contain the zero-point energy and the zero-point charge.

The existence of nontrivial identities (23) between bilinear products of ladder operators looks mysterious and there appears a natural question, what it means from the physical point of view. In the next section the physical bases for this fact will be considered, but some things from the formal point of view can be presented here.

The charge operator $Q$ is an analogue of an angular momentum $M$ and expresses symmetry under turn in the complex plane of the coordinate $q$. In the case of the angular momentum the rotation energy is expressed through the angular momentum as $H=M \omega$, where $\omega$ is angular velocity. Considering it, each of mutually chargeconjugate operators $Q, Q^{c}$ we will present as a sum of charge operators for two kinds of oscillators:

$$
Q=Q_{a}+Q_{\beta}, \quad Q^{c}=Q_{b}+Q_{\alpha} .
$$

where $Q_{a}=a^{*} a, Q_{\beta}=-\beta \beta^{*}, Q_{b}=b^{*} b, Q_{\alpha}=-\alpha \alpha^{*}$. Then it is possible to write the Hamiltonian (21) through these charge operators in the form:

$$
H=\left(Q_{a}-Q_{\beta}\right) \omega=\left(Q_{b}-Q_{\alpha}\right) \omega=H^{c}
$$

As the result, the charge conjugation symmetry conditions (13) transform into the relations between the charge operators for modes:

$$
\begin{aligned}
& Q_{a}-Q_{\beta}=Q_{b}-Q_{\alpha}, \\
& Q_{a}+Q_{\beta}=-Q_{b}-Q_{\alpha} .
\end{aligned}
$$

Then, after addition and subtraction of these two relations, we find:

$$
Q_{a}=-Q_{\alpha}, \quad Q_{\beta}=-Q_{b} .
$$

That is not only more compact form of the identities (23), but also adds them a certain physical meaning. Thus, the expressions for the observables take a form:

$$
\begin{aligned}
& H=H^{c}=Q_{a} \omega-Q_{b} \omega, \\
& Q=-Q^{c}=Q_{a}+Q_{b} .
\end{aligned}
$$

These relations reducing the relations between the observables to an "algebra of charges", show that a physical interpretation of vanishing of the zero-point energy for the charge-conjugation symmetric system oscillators can be related somehow by rotations, let even in a "charge space". Some additional arguments in favor of such treatment will be given in the next section. 


\section{About physical consequences of the charge conjugation symmetry of oscillators}

Lack of the zero-point energy for the system of harmonic oscillators with charge conjugation symmetry is unusual for the nonrelativistic systems. But generalization of the nonrelativistic harmonic oscillator model on relativistic fields is in fact purely formal and is far beyond on intuitive mechanical models. Therefore such new turnaround in consequences of the formalism, such as the lack of the zero-point energy in case specific to the relativistic systems, should be considered as a new prospect for investigations.

As examples of consequences which are specifically for the relativistic systems also, let us consider some new properties of systems of oscillators with such symmetry.

At quantization of harmonic oscillator's mechanical models, on the basis of intuitive understanding of the zero-point energy lies a restriction from the uncertainty relations. For understanding of the new situation from this point of view also, in Application 1 a generalization of these relations on systems with non-hermitian canonical variables, found earlier in [2], is presented:

$$
\left\langle q^{*} q\right\rangle\left\langle p^{*} p\right\rangle \geq \frac{1}{4}\left(i\left\langle q^{*} p^{*}-p q\right\rangle\right)^{2}=\frac{1}{4}\langle Q\rangle^{2} .
$$

It follows from them that if in a ground state of the system there is no zero-point charge and there is the charge conjugation symmetry, then $\langle Q\rangle=0$ and there are no ground state quantum fluctuations, i.e. the ground state evolves as a classical one. For a field it would mean that the vacuum of the field is like a classical external field which is not quantized (as a Coulomb field).

One more new consequence of charge-conjugation symmetry concerns the correlators. The commutator of operators

$$
\left[q\left(t^{\prime}\right), q^{*}(t)\right]=\frac{1}{2 m \omega}\left(\left[a, a^{*}\right] e^{-i \omega\left(t^{\prime}-t\right)}-\left[\beta, \beta^{*}\right] e^{i \omega\left(t^{\prime}-t\right)}\right)=\frac{-i}{m \omega} \sin \left[\omega\left(t^{\prime}-t\right)\right],
$$

as an odd function of time interval, disappears at equal times, which is natural.

More interesting is that at the formal approach the correlator of these variables in two moments of time (with causal restrictions) is odd function of time also:

$$
\begin{aligned}
\left\langle 0\left|q\left(t^{\prime}\right) q^{*}(t)\right| 0\right\rangle & =\frac{1}{2 m \omega}\left\langle 0\left|a a^{*} \theta\left(t^{\prime}-t\right) e^{-i \omega\left(t^{\prime}-t\right)}+\beta^{*} \beta \theta\left(t-t^{\prime}\right) e^{i \omega\left(t^{\prime}-t\right)}\right| 0\right\rangle= \\
= & \frac{1}{2 m \omega}\left[\theta\left(t^{\prime}-t\right) e^{-i \omega\left(t^{\prime}-t\right)}-\theta\left(t-t^{\prime}\right) e^{i \omega\left(t^{\prime}-t\right)}\right] .
\end{aligned}
$$

Here it is taken into account, that the ground states are defined as $a|0\rangle=0, b|0\rangle=0$, and, therefore, in accordance with identities (23), the relations

$$
\left\langle 0\left|\beta^{*} \beta\right| 0\right\rangle=\left\langle 0\left|\beta \beta^{*}-1\right| 0\right\rangle=\left\langle 0\left|b^{*} b-1\right| 0\right\rangle=-1 .
$$

take place. Such behavior of the propagator in the field theory would give instead of the standard causal propagator, which under construction is an even in time function, to the Pauli-Jordan function disappearing at zero time interval and outside the light cone. The construction of the even correlator will be considered below. It is interesting also the formal vanishing the ground state average:

$$
\left\langle 0\left|q\left(t^{\prime}\right)^{*} q(t)\right| 0\right\rangle=\frac{1}{2 m \omega}\left\langle 0\left|a^{*} a e^{i \omega\left(t^{\prime}-t\right)}+\beta \beta^{*} e^{-i \omega\left(t^{\prime}-t\right)}\right| 0\right\rangle=0,
$$

which leads to a "strong" normal ordering of operators in expressions for observables. 
In other side, since there remain only mutually charge-conjugate operators $a, b$ and the states are constructed also only by means of them, the theory with interactions also can be built on the basis of the same operators only. The "normal ordering" procedure then will be not a postulate, as before, but a consequence of the taking into account of the charge-conjugation symmetry conditions.

For clearing of physical sense of the nontrivial consequences of the chargeconjugation symmetry we will consider now combinations of this symmetry to other discrete symmetries which finally will lead to the crossing symmetry of amplitudes. There the charge conservation leads to two important differences from the oscillatory case.

By analogy to the linear oscillator at formation of matrix elements usually it is supposed that the operator $a^{*}$ increases to one a number of quanta in an initial state, and the operator $b$ reduces to one a number of the charge-conjugate quanta (antiquanta) in the same initial state of the system. At such definition of matrix elements the zero-point energy appears just as for the linear oscillator, and the charge conjugation symmetry will be broken.

On the one hand, arbitrary number of quanta of the linear oscillator can be created and destroyed without restrictions, obeying only the energy and momentum balance, whereas at propagation of a quantum with the conserved charge the joint worldlines of quanta and antiquanta must form a continuous trajectory.

On the other hand, the ground states defined as

$$
a|0\rangle=0, b|\overline{0}\rangle=0
$$

$(n, \bar{n}=0,1,2, \ldots-$ numbers of quanta and antiquanta), and the excited states:

$$
\begin{array}{ll}
a|n\rangle=\sqrt{n}|n-1\rangle, & a^{*}|n\rangle=\sqrt{n+1}|n+1\rangle, \\
b|\bar{n}\rangle=\sqrt{\bar{n}}|\bar{n}-1\rangle, & b^{*}|\bar{n}\rangle=\sqrt{\bar{n}+1}|\bar{n}+1\rangle,
\end{array}
$$

form two separately Hilbert spaces of states for quanta $|n\rangle$ and antiquanta $|\bar{n}\rangle$ which transform each other at the charge conjugation:

$$
|\bar{n}\rangle=C|n\rangle, \quad|n\rangle=C|\bar{n}\rangle \text {. }
$$

As the result each state of the system $|n, \bar{n}\rangle$ is a certain combination of states

from two Hilbert spaces. Which combination we take depends not on how it is convenient for us or customary, but from whether it is ensured at formation of matrix elements the conservation of the charge and crossing symmetry (the charge conjugation together with the hermitian conjugation). Thus, it concerns those processes which at the given initial and final states correspond to the alternatives the amplitudes of which should be summed.

Thus, if the matrix elements with operators $a, a^{*}$ are known, then corresponding matrix elements of alternatives with the operators $b, b^{*}$ it is necessary to be found by using the basic discrete symmetries of the theory - the time reversal and the charge conjugation - so that further they have not been broken, and their restrictions, such as the crossing symmetry, could be obeyed automatically.

By writing the operators of observables at first for the quanta:

$$
H_{a}=\omega a^{*} a, Q_{a}=a^{*} a,
$$

corresponding observables for the charge-conjugate quanta we can find by the charge conjugation of the operators of observables of quanta:

$$
H_{b}=C H_{a} C^{-1}=\omega b^{*} b, \quad Q_{b}=C Q_{a} C^{-1}=-b^{*} b .
$$


The total charge conjugation (anti) symmetric operators of observables then are the sum of these of two ones:

$$
H=H^{c}=H_{a}+H_{b}, \quad Q=-Q^{c}=Q_{a}+Q_{b} .
$$

For this expression the operators of coordinate we will rewrite through timedependent ladder operators including the time exponentials:

$$
q(t)=\frac{1}{\sqrt{2 m \omega}}\left[a(t)+b^{*}(t)\right], \quad q^{*}(t)=\frac{1}{\sqrt{2 m \omega}}\left[a^{*}(t)+b(t)\right]
$$

and we will consider the matrix elements from $a a^{*}$ :

$$
\left\langle 1 ; \Delta t, f\left|a^{*}(\Delta t)\right| 0\right\rangle\left[\left\langle 0\left|a(\Delta t) a^{*}(0)\right| 0\right\rangle\right]\langle 0|a(0)| 1 ; 0, i\rangle,
$$

describing the transition of an initial state into intermediate one with creation a new quantum at the moment $t_{i}=0$, its propagation during interval $\Delta t$ and transition into the final state at the moment $t_{f}=\Delta t$ with annihilation of this quantum. Alternative to (42) intermediate states with antiquanta at the same initial and final states give us the matrix elements of $b b^{*}$ :

$$
\left\langle 1 ;-\Delta t, f\left|a^{*}(-\Delta t)\right| 0\right\rangle\left[\left\langle\overline{0}\left|b(0) b^{*}(-\Delta t)\right| \overline{0}\right\rangle\right]\langle 0|a(0)| 1 ; 0, i\rangle
$$

They describe creation a pair at the moment $t_{f}=-\Delta t$ (a quantum in a final state and an antiquantum in the intermediate state), propagation of the antiquantum and its annihilation with the initial state quantum $t_{i}=0$ (crossing-diagram). In the given crossing transition the charge conjugation and the time interval reversal are made for the intermediate states. Thus $a^{*}(0)$ transfers into $b(0)$, and $a^{*}(\Delta t)$ into $b(-\Delta t)$ with cross transposition.

Thus, if operators of quanta in the intermediate state act on the right on the initial state, operators antiquanta - on the final state. As the result, there appear the crossingdiagrams with inverse order of time moments for the incoming of the initial and outcoming of the final states. The found identities (23), therefore, it is possible to consider as one of forms to express this feature - the crossing symmetry is expressed in the form of a modification of the operator ordering.

But, we can introduce another definition also - without changing of operator products - to change a definition of evaluation of matrix elements. Really, on the one hand, if from the expressions

$$
a^{*}(\Delta t) a(0) \rightarrow\left\langle n, \bar{n} ; \Delta t\left|a^{*}(\Delta t) a(0)\right| n, \bar{n} ; 0\right\rangle,
$$

we want to transfer to the crossing-symmetric to them, we obtain:

$$
b^{*}(0) b(-\Delta t) \rightarrow\left\langle n, \bar{n} ; \Delta t\left|b^{*}(0) b(-\Delta t)\right| n, \bar{n} ; 0\right\rangle .
$$

On the other hand, it is equivalent to the set of relationships:

$$
\begin{aligned}
b(-\Delta t) b^{*}(0) & \rightarrow b(-\Delta t)|n, \bar{n} ; 0\rangle\langle n, \bar{n} ; \Delta t| b^{*}(0)= \\
& =\left\langle n, \bar{n} ; \Delta t\left|b^{*}(0) b(-\Delta t)\right| n, \bar{n} ; 0\right\rangle .
\end{aligned}
$$

This means that as the recipe for practical evaluations it is possible to take the follow one: if the matrix element from the operators of particles is evaluated as $\left\langle f\left|a^{*}\right| n\right\rangle\langle n|a| i\rangle$, the corresponding matrix element for the crossing-symmetric to it should be evaluated as:

$$
\langle n|b| i\rangle\left\langle f\left|b^{*}\right| n\right\rangle=\left\langle f\left|b^{*}\right| n\right\rangle\langle n|b| i\rangle \text {. }
$$


The unusual identities (23) then will be symbolic expressions for the usual property of the crossing symmetry of matrix elements when the operators $\beta, \beta^{*}$ are the same $b, b^{*}$, but in the left-hand part of (47) even before transposition as in the right term. This means that:

$$
\begin{aligned}
& b\left(t^{\prime}\right)|0\rangle\langle 0| b^{*}(t)=\left\langle 0\left|b^{*} b\right| 0\right\rangle=0, \\
& b^{*}\left(t^{\prime}\right)|0\rangle\langle 0| b(t)=\left\langle 0\left|b(t) b^{*}\left(t^{\prime}\right)\right| 0\right\rangle .
\end{aligned}
$$

In such treatment the correlator formally becomes odd on time as in (32), and at taking of matrix elements by the rule (47) it will have the standard form:

$$
\left\langle 0\left|q\left(t^{\prime}\right) q^{*}(t)\right| 0\right\rangle=\frac{1}{2 m \omega}\left\langle 0\left|a a^{*} \theta\left(t^{\prime}-t\right) e^{-i \omega\left(t^{\prime}-t\right)}+b b^{*} \theta\left(t-t^{\prime}\right) e^{i \omega\left(t^{\prime}-t\right)}\right| 0\right\rangle,
$$

it also becomes even on time as well as in the former standard approach.

\section{Interactions of charge conjugation symmetric oscillators}

At considering of interactions of $C$-symmetric systems of oscillators we take the $C$-symmetric interaction potentials only. At a complex generalized coordinates $q$ and a potential $V\left(q^{*} q\right)$ there is $U(1)$ symmetry with the operator $U=\exp (i Q \alpha), \alpha=$ const. and corresponding charge is conserved: $\partial_{t} Q=0$. At a rotational symmetry $U(t)=\exp [i Q \alpha(t)]$ with a time-dependent parameter $\alpha(t)$ it is required an external gauge field $A_{\mu}=(\phi, \mathbf{A})$, transforming as $A_{\mu}{ }^{\prime}=A_{\mu}+\partial_{\mu} \alpha$ and leading to the covariant derivative $D_{t} \equiv \partial_{t}-i g \phi$ with $g$ as a coupling constant.

The minimal form of a gauge-invariant Lagrangian looks like:

$$
\begin{aligned}
L & =m\left(D_{t} q\right)^{*}\left(D_{t} q\right)-V= \\
& =m\left(\partial_{t} q\right)^{*}\left(\partial_{t} q\right)+g Q \phi+m g^{2} \phi^{2} q^{*} q-V,
\end{aligned}
$$

Generalized momenta $\pi^{*}(t)=m\left(D_{t} q\right)$ and $\pi(t)=m\left(D_{t} q\right)^{*}$ at quantization should commutate each other, but then the velocities $\partial_{t} q$ and $\left(\partial_{t} q\right)^{*}$ do not commutate:

$$
\begin{aligned}
& {\left[\pi(t), \pi^{*}(t)\right]=0,} \\
& {\left[\partial_{t} q(t), \partial_{t} q^{*}(t)\right]=\frac{2}{m} g \phi M,}
\end{aligned}
$$

where the effective "angular momentum" $M$ is defined as usually:

$$
M=i\left(q p-q^{*} p^{*}\right)=q_{1} p_{2}-q_{2} p_{1}=-(Q+1) .
$$

As the result, the choice of ordering of product of velocities in the quantum Lagrangian is an additional physical hypothesis, defining presence or lack of a zero-point energy and a zero-point charge.

For a system with the harmonic potential $V=k q^{*} q$ the Lagrangian (50) in general case leads to the zero-point energy and the zero-point charge, which corresponds to vibrational modes of independent oscillators. In the systems with the same potential and the exact charge-conjugation symmetry there will be no zero-point energy (and a zero-point charge). 
At $C$-symmetry of an interaction potential: $V=V_{c}$, where $V_{c}=C V C^{-1}$, the full Hamiltonian also will be $C$-symmetric and obeys (for the potentials, not changing a charge operator) the condition:

$$
H=\omega\left(a^{*} a+\beta \beta^{*}\right)+g Q \phi+V=\omega\left(b^{*} b+\alpha \alpha^{*}\right)+g Q^{c} \phi^{c}+V_{c}=H_{c} .
$$

Here contributions of the interaction potentials in both sides of equality are the same. As a result, the symmetry condition for the full Hamiltonian is reduced to the former one for the unperturbed Hamiltonian and the earlier obtained operator identities (23) are remain the same at the presence of the $C$-symmetric interactions.

For $C$-symmetric contributions the terms of any order of perturbation theory also will be $C$-symmetric. Really, thanks to (53) energies of the C-conjugate levels are identical, and the matrix elements are related across the $C$-symmetry conditions:

$$
\begin{aligned}
& \left\langle n_{+}|V| n_{+}\right\rangle=\left\langle n_{+}\left|C^{-1} V_{c} C\right| n_{+}\right\rangle=\left\langle n_{-}\left|V_{c}\right| n_{-}\right\rangle, \\
& \left\langle n_{+}|V| m_{ \pm}\right\rangle\left\langle m_{ \pm}|V| n_{+}\right\rangle=\left\langle n_{-}\left|V_{c}\right| m_{\mp}\right\rangle\left\langle m_{\mp}\left|V_{c}\right| n_{-}\right\rangle, \ldots
\end{aligned}
$$

Thus, such contributions are reduced to the symmetry condition (53), leaving in equality only the contributions of the unperturbed Hamiltonian and, consequently, the identities (23) are hold at taking into account of contributions of higher orders of a perturbation theory also.

The important problem is whether energy of a ground state changes at inserting of interactions, when there is no zero-point energy in the unperturbed Hamiltonian. Since in the charge operator $Q$ the ladder operators enter in the normal-ordered form, the interactions do not contribute to the vacuum expectations from the full charge operator in perturbation theory.

In the first order of perturbation theory nonzero expectations from (52) for ground state can be only from the terms proportional to the external field. But, at first, these contributions disappear at removal of an external field, and secondly, under the $C$ symmetry condition they disappear at lack of external field also.

Really, in perturbation theory first order nonvanishing contributions to the ground state energy from the Hamiltonian look like:

$$
\langle 0|\tilde{H}| 0\rangle=\phi\left\langle 0\left|\tilde{Q}_{\phi}\right| 0\right\rangle=\phi^{2}\left\langle 0\left|\left(q^{*} q+q_{c}^{*} q_{c}\right)\right| 0\right\rangle .
$$

In ladder operators representation we see that at using the operator identities the matrix element vanishes:

$$
\left\langle 0\left|\left(q^{*} q+q_{c}^{*} q_{c}\right)\right| 0\right\rangle=\frac{1}{2 \omega}\left\langle 0\left|\alpha \alpha^{*}+\beta \beta^{*}\right| 0\right\rangle=\frac{1}{2 \omega}\left\langle 0\left|a^{*} a+b^{*} b\right| 0\right\rangle=0 .
$$

As we see, the vanishing of matrix elements is related with the normal-ordered form of the bilinear operator products. Since the full number of operators is always even, the higher orders of perturbation theory will give similar results and their contribution to the ground state energy also disappears.

\section{The problem of negative norm for negative-frequency states and its solution}

A state, charge-conjugate to given state, can formally be treated as the latter one evolving backward in time, which is often easier and more convenient for description.

In quantum mechanics the time reversal operation $T$ can be introduced be two ways. In the first one, accepted in the nonrelativistic theory, at the time reversal $t \rightarrow-t$ the sign on energy remains unchanged $H \rightarrow H$, but then $T$ is described by an antiunitary 
operator in a combination with additional hermitian conjugation of matrix elements (which is in fact a hidden changing the sign on energy).

In the second definition of $T$, often using in the relativistic theory, it is a part of a spacetime inversion $x^{\mu} \rightarrow-x^{\mu}(P T)$ described by a unitary operator, and thus the system's 4-momentum changes a sign: $p_{\mu} \rightarrow-p_{\mu}$, i.e. a sign on energy changes also. In the relativistic theory a natural and more convenient one is this definition of the time reversal in the combination with $C P$-symmetry. For states of particles with $-p_{\mu}$ it is admitted formally only backward in time evolution and then they are interpreted from the physical point of view as forward in time evolving states of antiparticles with $+p_{\mu}$. This treatment allows then to construct the causal propagators of particles.

Besides, it allows interpreting an initial state of the particle $-p_{\mu}$ as a final state of the antiparticle with $+p_{\mu}$. This is widely using in particle physics a crossing symmetry property of processes when the changing of a sign of particle's 4-momentum in a diagram or rotation of its line around a vertex with transfer into opposite light cone transforms it into an antiparticle (and contrary).

Further in the paper it will be taken as a basis this second form of the time reversal as a part of 4-inversion $P T$ naturally coincided with $C P T$-symmetry in general and with the crossing symmetry in particular cases. Thus, at the time reversal $t \rightarrow-t$ it will be changed both a sign on frequency $\omega \rightarrow-\omega$ and a sign on energy $H \rightarrow-H$ which for the harmonic oscillator's quanta directly follows from the formula for energy.

In most of standard formulas for the harmonic oscillator the frequency appears in the form of products - with mass $(m \omega)$, with time $(\omega t)$ or quadratically, and only in the formula for energy enters linearly and determines a sign of energy. From expressions for the harmonic oscillator wave functions follows that a ground state wave function

$$
|0\rangle=\psi_{0}(x)=\left(\frac{m \omega}{\pi}\right)^{1 / 4} e^{-m \omega x^{2} / 2} .
$$

remains as a real and unincreasing one on spatial infinity only at a simultaneous changing of signs of frequency and mass $\omega \rightarrow-\omega, m \rightarrow-m$, when their product does not change a sign:

$$
m_{ \pm} \omega_{ \pm}=m \omega,
$$

where $m>0, \omega>0$ and $m_{ \pm}= \pm m \omega_{ \pm}= \pm \omega$. The wave functions will not vary if $\omega_{ \pm} \cdot( \pm t)=\omega t$. The commutators of the ladder operators

$$
\left[a_{ \pm}, a_{ \pm}^{*}\right]=1 \text {. }
$$

do not depend on frequency and mass and, consequently, they also will not vary.

At former approaches to treatment of states of the oscillator there it has been implicitly suggested that a positive mass in negative-frequency states is positive. As the result, this led to the negative sign in the commutator instead of (59) and further to the negative norm of states.

On the one hand, this suggestion is unphysical since the mass in the relativistic theory is nothing but as energy in the rest frame only and, consequently, the states with the positive mass should not evolve backward in time, as well as the states with the negative mass should not evolve forward in time. On the other hand, as soon as we will consider signs of mass, frequencies and energies as identical, commutators will be 
always positive as in (59) and the norm of negative-energy states also will remain positive.

Thus, the one of "virus-hypotheses" was a suggestion that the negative energy states, going inversely in time, have a positive mass. By excluding this implicit hypothesis and taking signs of mass and energy coinciding with a sign on frequency, we come to the consistent theory of the oscillator, the basic formulas of which will take the following form.

The Lagrangians of the linear harmonic oscillators, having two kinds of states of opposite signs on the circular frequency $\omega_{ \pm}$, with real (generalized) coordinates $x_{ \pm}$and masses of oscillating particle $m_{ \pm}$:

$$
L_{ \pm}=\frac{m_{ \pm}}{2}\left[\left(\partial_{t} x_{ \pm}\right)^{2}-\omega^{2} x_{ \pm}^{2}\right]
$$

lead to the momenta $p_{ \pm}=m_{ \pm} \partial_{t} x_{ \pm}$, the Hamiltonians:

$$
H_{ \pm}=\frac{1}{2 m_{ \pm}}\left[p_{ \pm}^{2}+(m \omega)^{2} x_{ \pm}^{2}\right]
$$

and the equations of motion:

$$
\partial_{t}^{2} x_{ \pm}+\omega^{2} x_{ \pm}=0
$$

The solutions of the equations of motion lead to the frequency decomposition of the coordinates and momenta:

$$
x_{ \pm}=\frac{1}{\sqrt{2 m \omega}}\left(a_{ \pm} e^{-i \omega t}+a_{ \pm}^{*} e^{i \omega t}\right), \quad p_{ \pm}=\frac{-i m \omega}{\sqrt{2 m \omega}}\left(a_{ \pm} e^{-i \omega t}-a_{ \pm}^{*} e^{i \omega t}\right) .
$$

At such representation of the operators of coordinate the first term reduces a number of quanta by unity in an initial state, and second - in a final state, and on the contrary, the first term increases number of quanta in a final state, and second in the initial. Ladder operators in (63) are defined as:

$$
a_{ \pm}=\frac{e^{i \omega t}}{\sqrt{2 m \omega}}\left(m \omega x_{ \pm}+i p_{ \pm}\right), \quad a_{ \pm}^{*}=\frac{e^{-i \omega t}}{\sqrt{2 m \omega}}\left(m \omega x_{ \pm}-i p_{ \pm}\right) .
$$

At quantization the commutators $i\left[p_{ \pm}, x_{ \pm}\right]=1$ lead to the commutators for the ladder operators presented in (59).

Thus, the operators of opposite sign frequency states mutually commutate since they act on different state spaces. These states of both types of oscillators are defined by acting of corresponding ladder operators on their ground and excited states $\left|n_{ \pm}\right\rangle$:

$$
a_{ \pm}\left|n_{ \pm}\right\rangle=\sqrt{n_{ \pm}}\left|n_{ \pm}-1\right\rangle, \quad a_{ \pm}^{*}\left|n_{ \pm}\right\rangle=\sqrt{n_{ \pm}+1}\left|n_{ \pm}+1\right\rangle, \ldots
$$

where $n_{ \pm}=0,1,2, \ldots$ are numbers of positive - and negative-frequency quanta. The ladder operators act on the wave functions in the occupation numbers representation. From the normalized wave function of ground states:

$$
\left|0_{ \pm}\right\rangle \rightarrow \psi_{0}\left(x_{ \pm}\right)=\left(\frac{m \omega}{\pi}\right)^{1 / 4} e^{-m \omega x_{ \pm}^{2} / 2}
$$

by using (65), we can construct the wave functions of excited states. The Hamiltonian (61) and the number operator of quanta $N_{ \pm}$, as the result, take the form:

$$
H_{ \pm}=\omega_{ \pm}\left(N_{ \pm}+\frac{1}{2}\right), \quad N_{ \pm}=a_{ \pm}^{*} a_{ \pm}
$$


Thus, the standard theory of the harmonic oscillator extended with inserting of negative-frequency states, is self-consistent if we always take the same signs of mass and energy. The ground states are defined as lowest energy state for the positive-frequency sector and highest energy state for the negative-frequency sector.

Let us redefine coordinates and momenta so that further the dimensional constant - a value of mass $m=\left|m_{ \pm}\right|$will not figure in formulas:

$$
\tilde{x}_{ \pm}=\sqrt{m} x_{ \pm}, \quad \tilde{p}_{ \pm}=p_{ \pm} / \sqrt{m} .
$$

The Lagrangian, momenta and the Hamiltonian of the system then take the form:

$$
\begin{gathered}
L_{ \pm}= \pm \frac{1}{2}\left[\left(\partial_{t} \tilde{x}_{ \pm}\right)^{2}-\omega^{2} \tilde{x}_{ \pm}^{2}\right], \\
H_{ \pm}= \pm \frac{1}{2}\left(\tilde{p}_{ \pm}^{2}+\omega^{2} \tilde{x}_{ \pm}^{2}\right), \quad \tilde{p}_{ \pm}= \pm \partial_{t} \tilde{x}_{ \pm} .
\end{gathered}
$$

Notice the changing a sign of negative-frequency contributions.

The commutators and equations of motion thus will not vary, and the frequency decomposition takes the form:

$$
\tilde{x}_{ \pm}=\frac{1}{\sqrt{2 \omega}}\left(a_{ \pm} e^{-i \omega_{ \pm} t}+a_{ \pm}^{*} e^{i \omega_{ \pm} t}\right), \quad \tilde{p}_{ \pm}=\frac{-i \omega}{\sqrt{2 \omega}}\left(a_{ \pm} e^{-i \omega_{ \pm} t}-a_{ \pm}^{*} e^{i \omega_{ \pm} t}\right),
$$

where the ladder operators $a_{ \pm}, a_{ \pm}^{*}$ remained unchanged, but expressed in the form:

$$
a_{ \pm}=\frac{e^{i \omega_{ \pm} t}}{\sqrt{2 \omega}}\left(\omega x_{ \pm}+i p_{ \pm}\right), \quad a_{ \pm}^{*}=\frac{e^{-i \omega_{ \pm} t}}{\sqrt{2 \omega}}\left(\omega x_{ \pm}-i p_{ \pm}\right) .
$$

The commutators (59) are positive for states of both signs of frequency and the norm of these states is also positive defined.

Above the harmonic oscillators with opposite signs of frequency (and masses) were considered separately. Let us consider a model including simultaneously the states of both sign of frequency. The negative-frequency state from the physical point of view describes an oscillating antiparticle in terms of variables of a particle - in the form of an inversely in time evolving particle.

Then the Lagrangian and Hamiltonian of the system look like:

$$
\begin{gathered}
L=\frac{m_{+}}{2}\left[\left(\partial_{t} x_{+}\right)^{2}-\omega^{2} x_{+}^{2}\right]+\frac{m_{-}}{2}\left[\left(\partial_{t} x_{-}\right)^{2}-\omega_{-}^{2} x_{-}^{2}\right], \\
H=\frac{1}{2 m_{+}}\left[p_{+}^{2}+(m \omega)^{2} x_{+}^{2}\right]+\frac{1}{2 m_{-}}\left[p_{-}^{2}+(m \omega)^{2} x_{-}^{2}\right] .
\end{gathered}
$$

At redefining of the coordinates and momenta as in (68) with elimination of the value of mass, the Lagrangian and the Hamiltonian of the system take the form:

$$
\begin{gathered}
L=\frac{1}{2}\left[\left(\partial_{t} \tilde{x}_{+}\right)^{2}-\omega^{2} \tilde{x}_{+}^{2}\right]-\frac{1}{2}\left[\left(\partial_{t} \tilde{x}_{-}\right)^{2}-\omega^{2} \tilde{x}_{-}^{2}\right], \\
H=\frac{1}{2}\left(\tilde{p}_{+}^{2}+\omega^{2} \tilde{x}_{+}^{2}\right)-\frac{1}{2}\left(\tilde{p}_{-}^{2}+\omega^{2} \tilde{x}_{-}^{2}\right) .
\end{gathered}
$$

Then the Hamiltonian and the number operator take the form:

$$
H=\omega_{+}\left(N_{+}+\frac{1}{2}\right)+\omega_{-}\left(N_{-}+\frac{1}{2}\right),
$$

and in $H$ there are zero-point energies of two kinds of oscillators. 
Thus, at redefining of dynamical variables (68) the mass parameter formally does not figure in formulas, but nevertheless the sign of mass remains and it changes a sign of negative-frequency contributions in the Lagrangian and Hamiltonian. This property allows us to introduce the negative-frequency modes into the field theories also, where field's "oscillators" appear without the mass parameter.

\section{Conclusion}

The observables of charge conjugation symmetric systems of harmonic oscillators can be expressed through two kinds of ladder operators which are charge-conjugate to each other and the $C$-symmetry conditions are obeyed exactly. Then such system does not contain the zero-point energy and zero-point charge.

The $C$-symmetric interactions leave unchanged and equal to zero the ground state energy in all orders of perturbation theory. The crossing symmetry of states allows to understand obtained operator identities and to find relations between matrix elements.

At representation of states of antiquanta as states of quanta with the negative frequency and energy, the mass also should be negative. At such correct approach the norm of the negative frequency states remains positive and the theory is consistent.

\section{Acknowledgments}

Last five years the ideas and results presented in the paper have been subjected numerous heated discussions and partially thanks them have reached some completeness and clearness. As any new method, which works, but while unclear why, they were subjected a destructive criticism with totally ignoring the results. Since usually such good luck as a combination of a right description with a correct explanation is infrequent, I interested only by the analysis of consequences and correctness of description and grateful to all those who had patience to listen results which I can derive, but still cannot explain.

\section{Appendices.}

\section{The uncertainty relations for non-hermitian variables}

The standard Heisenberg's uncertainty relations are valid only for systems with hermitian operators of canonical variables. In those systems, where operators of the observable are constructed as hermitian products of non-hermitian canonical variables, a generalization of these relations is required.

Let us take $\langle q\rangle=\left\langle q^{*}\right\rangle=0$ and consider relations between the expectations from the bilinear combinations $\left\langle q^{*} q\right\rangle,\left\langle p^{*} p\right\rangle,\left\langle q^{*} p^{*}\right\rangle$ and $\langle p q\rangle$. An inequality:

$$
\left\langle\left(\lambda q^{*}-\frac{i}{\hbar} p\right)\left(\lambda q+\frac{i}{\hbar} p^{*}\right)\right\rangle \geq 0,
$$

where $\lambda$ is an arbitrary real number, at a distribution of brackets gives:

$$
\lambda^{2}\left\langle q^{*} q\right\rangle+\lambda \frac{i}{\hbar}\left\langle q^{*} p^{*}-p q\right\rangle+\frac{1}{\hbar^{2}}\left\langle p^{*} p\right\rangle \geq 0
$$

This inequality is satisfied at arbitrary real valued $\lambda$ only at:

$$
\left\langle q^{*} q\right\rangle\left\langle p^{*} p\right\rangle \geq \frac{1}{4}\left(i\left\langle q^{*} p^{*}-p q\right\rangle\right)^{2}=\frac{1}{4}\langle Q\rangle^{2} .
$$

In case of complex system, where charge eigenvalues are quantized: 


$$
\langle Q\rangle=i\left\langle q^{*} p^{*}-p q\right\rangle=\left\langle[q, p]_{*}\right\rangle=\hbar n_{( \pm)},
$$

where $n_{ \pm}=0, \pm 1, \pm 2, \ldots$, the relations (80) take a form:

$$
\left\langle q^{*} q\right\rangle\left\langle p^{*} p\right\rangle \geq \frac{\hbar^{2}}{4} n_{ \pm}^{2}
$$

The relations $(80)$ - (82) are the generalizations of uncertainty relations for the systems with non-hermitian canonical variables [1]. The expression for non-zero mean values is obtained by replacements $q^{\prime}=q-\langle q\rangle, \quad p^{\prime}=p-\langle p\rangle$.

At exact charge conjugation symmetry the ground state has no charge: $\langle Q\rangle_{0}=0$ and from (80) follows:

$$
\left\langle q^{*} q\right\rangle_{0}\left\langle p^{*} p\right\rangle_{0} \geq 0
$$

In particular, for relativistic fields with non-hermitian canonical variables and $C$ symmetry, the vacuum does not contain a zero-point energy [1].

Since the expression on the right hand of (80) can be represented through angular momentum eigenvalues also, the inequality takes a form:

$$
\left\langle q^{*} q\right\rangle\left\langle p^{*} p\right\rangle \geq \frac{\hbar^{2}}{4}(1+\langle M\rangle)^{2} .
$$

For real valued canonical variables $(l=0)\langle M\rangle=0$ the expression $q^{*} p^{*}-p q$ turns to the usual commutator $(q p-p q)=i \hbar$, leading to the standard Heisenberg's uncertainty relation.

\section{Canonical formalism for systems with complex variables}

At the standard definition of Poisson brackets for two pairs of canonical variables, as a rule, two brackets have been considered only:

$$
\{A, B\}_{P B} \equiv \frac{\partial A}{\partial p} \frac{\partial B}{\partial q}-\frac{\partial A}{\partial q} \frac{\partial B}{\partial p}, \quad\{A, B\}_{P B^{*}}=\frac{\partial A}{\partial p^{*}} \frac{\partial B}{\partial q^{*}}-\frac{\partial A}{\partial q^{*}} \frac{\partial B}{\partial p^{*}} .
$$

Each of them is invariant under one of two groups of canonical transformations: $(p, q) \rightarrow(P, Q),\left(p^{*}, q^{*}\right) \rightarrow\left(P^{*}, Q^{*}\right)$.

In fact in such complex systems it is possible to consider mixed brackets also:

$$
\begin{gathered}
\{A, B\}_{*} \equiv \frac{\partial A}{\partial p^{*}} \frac{\partial B}{\partial q^{*}}-\frac{\partial A}{\partial q} \frac{\partial B}{\partial p}, \quad\{A, B\}_{m 1} \equiv \frac{\partial A}{\partial p} \frac{\partial B}{\partial q}-\frac{\partial A}{\partial q^{*}} \frac{\partial B}{\partial p^{*}}, \\
\{A, B\}_{m 2} \equiv \frac{\partial A}{\partial p} \frac{\partial B}{\partial q}-\frac{\partial A}{\partial p^{*}} \frac{\partial B}{\partial q^{*}}, \quad\{A, B\}_{m 3} \equiv \frac{\partial A}{\partial q} \frac{\partial B}{\partial p}-\frac{\partial A}{\partial q^{*}} \frac{\partial B}{\partial p^{*}} .
\end{gathered}
$$

In case of real generalized coordinates first two mixed brackets transfer into usual Poisson brackets, and two last, changing a sign at interchanging $q \rightarrow q^{*}, p \rightarrow p^{*}$, disappear. Six kinds of brackets (85) and (86) are related among themselves by three identities:

$$
\begin{aligned}
& \{A, B\}_{*}+\{A, B\}_{m 1}=\{A, B\}_{P B}+\{A, B\}_{P B^{*}}, \\
& \{A, B\}_{m 2}=\{A, B\}_{*}-\{A, B\}_{P B^{*}}, \quad\{A, B\}_{m 3}=\{A, B\}_{P B^{*}}-\{A, B\}_{m 1} .
\end{aligned}
$$

therefore, three of them are independent only, as which we can choose two standard ones, and one of the mixed brackets, for example $\{A, B\}_{*}$. 
30 Zakir Z. (2011) Theoretical Physics, Astrophysics and Cosmology, 6, 14; TPAC: 3900-021 v2.

From the physical point of view this third independent bracket is related to the canonical invariant related to a rotational symmetry on a complex plane of generalized coordinates $q$ and, consequently, this invariant is an angular momentum $M$ at rotations in real space or a charge $Q$ at rotations in space of complex variables. Thus, a physical observable should be expressed through (bilinear) real combinations of complex coordinates and momenta.

For the generalized coordinates and momenta then we have:

$$
\begin{gathered}
\left\{p_{i}, q_{j}\right\}_{P B}=\left\{p_{i}^{*}, q_{j}^{*}\right\}_{P B^{*}}=\delta_{i j},\left\{p_{i}, q_{j}\right\}_{*}=\left\{p_{i}, q_{j}\right\}_{m 1}=-\left\{p_{i}^{*}, q_{j}^{*}\right\}_{m 1}=\delta_{i j} \\
\left\{p_{i}, q_{j}\right\}_{*}=\left\{p_{i}, q_{j}\right\}_{m 1}=-\left\{p_{i}^{*}, q_{j}^{*}\right\}_{m 1}=\delta_{i j} .
\end{gathered}
$$

Besides, to usual relations:

$$
\{A, q\}_{P B}=\frac{\partial A}{\partial p}, \quad\{p, B\}_{P B}=\frac{\partial B}{\partial q},\left\{A, q^{*}\right\}_{P B^{*}}=\frac{\partial A}{\partial p^{*}}, \quad\left\{p^{*}, B\right\}_{P B^{*}}=\frac{\partial B}{\partial q^{*}},
$$

new ones are added:

$$
\begin{gathered}
\{A, q\}_{*}=\frac{\partial A}{\partial p}, \quad\{p, B\}_{*}=\frac{\partial B}{\partial q},\left\{q^{*}, B\right\}_{*}=-\frac{\partial B}{\partial p^{*}},\left\{A, p^{*}\right\}_{*}=-\frac{\partial A}{\partial q^{*}}, \\
\{q, B\}_{m 1}=-\frac{\partial B}{\partial p},\{A, p\}_{m 1}=-\frac{\partial B}{\partial q},\left\{A, q^{*}\right\}_{m 1}=\frac{\partial A}{\partial p^{*}},\left\{p^{*}, B\right\}_{m 1}=\frac{\partial B}{\partial q^{*}} .
\end{gathered}
$$

These relations can be used at integration of equations of motion.

Since the standard commutators $[A, B]$ are quantum analogues of the standard Poisson brackets $\{A, B\}_{P B}$, the generalized angular momentum (charge) $Q$ is quantum analogue of the mixed bracket $\{A, B\}_{*}$. By introducing a mixed commutator corresponding to this bracket $[A, B]_{*}$ :

$$
[A, B]_{*}=i\left(A^{*} B^{*}-B A\right),
$$

the charge operator can be expressed through it in the form:

$$
Q=[q, p]_{*} \text {. }
$$

For a real valued generalized coordinates $Q$ turns to the standard commutator:

$$
Q \rightarrow i(q p-p q)=[q, p] \text {. }
$$

\section{References}

1. Zakir Z. (2006) Theor. Phys., Astrophys. and Cosmol., 1, 1, 1; 1, 1, 11.

2. Zakir Z. (2007) Theor. Phys., Astrophys. and Cosmol., 2, 2, 9; arXiv:0705.0899. 